\title{
Complex changes of circadian proteins expression in inflammatory bowel disease
}

\author{
Mosna $\mathrm{K}^{1,2}$, Janega $\mathrm{P}^{1,2,3}$, Sedlak $\mathrm{J}^{4}$, Babal $\mathrm{P}^{1,2}$ \\ Institute of Pathological Anatomy, Faculty of Medicine, Comenius University, Bratislava, Slovakia. \\ giertlova2@uniba.sk
}

\section{ABSTRACT}

OBJECTIVES AND BACKGROUND: Recently, a possible role of circadian system in the pathogenesis of various gastrointestinal disorders gained an attention. The association of circadian system with immune system activity and reciprocal connection with intestinal microbiota indicate possible links with inflammatory bowel diseases (IBD).

METHODS: The retrospective study provided a semiquantitative immunohistochemical analysis of the expression of 8 core circadian proteins (BMAL1, BMAL2, PER1, PER2, PER3, CLOCK, NPAS2 and TIMELESS) in the epithelial cells of intestinal mucosa in 24 patients with Crohn's disease (CD) and 26 patients with ulcerative colitis (UC). Samples from patients without history of IBD served as the control. The BMAL1 protein expression in intramucosal inflammatory cells was explored as well.

RESULTS: The expression of 5 core circadian proteins (BMAL1, PER1, PER3, TIMELESS and NAPS2) was decreased in mucosal epithelium of patients with IBD in comparison with the control samples, whereas the expression of BMAL1 and PER1 was more noticeably decreased in UC patients and PER3, TIMELESS and NPAS2 in CD patients. There was a decreased BMAL1 expression in intramucosal inflammatory cells of IBD patients.

CONCLUSION: Decreased core circadian proteins expression in colonic mucosa and in intramucosal inflammatory cells of IBD patients indicated a circadian rhythm deregulation as contributing factor in the development of IBD. To our knowledge, this is so far the most extensive immunohistochemical analysis performed on the samples of IBD patients evaluating the changes in circadian protein expression in the intestinal mucosa (Tab. 1, Fig. 2, Ref. 31). Text in PDF www.elis.sk

KEY WORDS: circadian rhythm deregulation, circadian rhythm proteins, inflammatory bowel disease, ulcerative colitis, Crohn disease, immunohistochemistry.

\begin{abstract}
Abbreviations: IBD - Inflammatory bowel disease, CD - Crohn's disease, UC - ulcerative colitis, GIT - gastrointestinal tract, SCN - nucleus suprachiasmaticus, QS - Quick score
\end{abstract}

\section{Introduction}

The term inflammatory bowel disease (IBD) encompasses conditions involving chronic, relapsing inflammation of gastrointestinal tract (GIT) and comprises mainly two pathological entities: Crohn's disease (CD) and ulcerative colitis (UC). IBD was

${ }^{1}$ Institute of Pathological Anatomy, Faculty of Medicine, Comenius University, Bratislava, Slovakia, ${ }^{2}$ Institute of Pathological Anatomy, University Hospital Bratislava, Bratislava, Slovakia, ${ }^{3}$ Centre of Experimental Medicine, Slovak Academy of Sciences, Bratislava, Slovakia, and ${ }^{4}$ Cancer Research Institute, Biomedical Research Center, Slovak Academy of Sciences, Bratislava, Slovakia

Address for correspondence: K. Mosna, MD, Institute of Pathological Anatomy, University Hospital Bratislava and Faculty of Medicine, Comenius University, Sasinkova 4, SK-811 08 Bratislava, Slovakia. Phone: +421904986521

Acknowledgements: This work was supported by the Slovak Research and Development Agency (grant APVV-14-0318) traditionally perceived as a disease of the Western world, however, over the past decades, the incidence in the newly industrialized countries worldwide started to show a rising tendency, turning IBD into an emerging global health challenge affecting millions $(1,2)$

Even though the IBD is currently widely studied and vast research is continuously providing new insights, the exact etiology and pathogenesis remain unknown. According to the current hypotheses, IBD is the result of an exaggerated uncontrolled immune response triggered in genetically susceptible hosts with a various participation of environmental or infectious factors and abnormal intestinal flora $(2,3)$. The latest research suggests that sleep and melatonin might play an important role in the development of IBD, mainly through the modification of the immune system and gut microbiota, implying a possible role of circadian rhythms in the pathogenesis of this disorder $(4,5)$.

Circadian rhythms are endogenously generated rhythms with a periodicity of approximately 24 hours that are necessary for the synchronization of endogenous processes with the exogenous environment (6). In humans, there are two types of circadian oscillators - central and peripheral, consisting of numerous autonomous cellular oscillators. Peripheral oscillators, which are located throughout the body, are regulated by signals received form central oscil- 


\section{5-241}

lator. Central oscillator, located in nc. suprachiasmaticus (SCN) in anterior hypothalamus, generates autonomous signals, which are regulated by environmental cues, mainly by external cycles of light and darkness (7). Peripheral oscillators are also regulated by indirect cues that arise as a result of circadian behaviour, like body temperature and food intake. They are responsible for rhythmic transcription of around 10-20\% of genes in peripheral tissues, which play a role in the regulation of tissue-specific functions (8).

Circadian rhythms are on the molecular level generated by clock genes through a series of intertwined autoregulated positive and negative transcription/translation feedback loops. The most important clock genes so far recognized include Per1, Per2, Per3 (period), Bmal1 (Arntl) (brain and muscle aryl-hydrocarbon receptor nuclear translocator-like), Clock (circadian locomotor output cycles kaput), Cry1, Cry2 (cryptochrome), Npas2 (neuronal PAS domain), Timeless, Tipin (timeless interacting protein) and CK1epsilon. The basic principle of circadian rhythms generation is a successive cyclical activation of genes, whereby the first gene in the sequence is regulated by the last gene, creating an autoregulated feedback loop with the duration of one cycle approximately 24 hours (9).

Rhythmic oscillations of various GIT processes have been known for decades. In recent years, numerous components of the molecular circadian system have been identified within the GIT. Circadian expression of clock genes is localized mainly in neurons of myenteric plexus and in the epithelial cells of crypts, including Paneth cells and stem cells (10).

Since almost all human physiological functions follow 24-hour rhythms, their deregulation, referred to as chronodisruption, is considered to be an important factor contributing to the development of a spectrum of pathological disorders (4). Chronodisruption might be evoked by gene polymorphisms, aging or environmental factors like an exposition to light during the night $(11,12)$. Several studies demonstrated a relationship between the deregulation of circadian rhythms and gastrointestinal neoplasms (13), nevertheless, there is only a limited amount of data about the potential connection of IBD and circadian system disruption. Up to date information anticipate a possible contribution of circadian system deregulation to the development of IBD by several mechanisms, including the impact of circadian rhythms on immune system $(14,15)$, and intestinal microflora (16). Molecular mechanisms linking circadian rhythms and IBD are so far not fully understood, however, ongoing studies, including ours, try to contribute in clarifying this connection by pointing out differences in the regulation of clock genes in healthy versus inflamed intestinal tissue.

\section{Materials and methods}

\section{Patient samples and ethical considerations}

In this retrospective study, the changes of expression of circadian proteins were evaluated on formalin-fixed paraffin-embedded samples of intestinal mucosa from the patients with IBD. The samples were obtained from archives of the Institute of Pathological Anatomy, Faculty of Medicine, Comenius University in Bratislava and University Hospital Bratislava after the St. Elizabeth Cancer
Institute ethics committee approval. Overall, 50 patients with diagnosed IBD between the years 2012 and 2017 were included in the study, of which 26 were diagnosed with CD and 24 with UC. All the included intestinal samples showed classic histological signs of given diagnoses in conformity with ESP (European Society of Pathology) and ECCO (The European Crohn's and Colitis Organisation) histopathology guidelines (17). 25 control samples were obtained mainly from large intestine resection specimens of patients with colorectal carcinoma, and the examined tissue was separated from pathological affection by 10 or more centimetres. Patients samples were obtained between 8:00 a.m. and 1:00 p.m.

\section{Immunohistochemistry}

Collected samples were cut to $5 \mu \mathrm{m}$ slices, deparaffinized and after antigen retrieval in citrate buffer, the slides were incubated with primary antibodies against 8 core circadian proteins in following dilutions: BMAL1 (NB100-2288, Novus Biological) 1:300; BMAL2 (C-11, sc-365469, Santa Cruz) 1:100; NPAS2 (NBP188612, Novus Biological) 1:100; TIMELESS (ab72458, Abcam) 1:200; PER1 (ab3443, Abcam) 1:100; PER2 (ab200388, Abcam) 1:200; PER3 (ab224594, Abcam) 1:500; CLOCK (ab134165, Abcam) 1:2000. The reaction was developed by EnVision kit and diaminobenzidine chromogen (Agilent, Santa Clara, CA, USA) according to the manufacturer's instructions.

\section{Immunohistochemical staining evaluation}

Staining was evaluated semi quantitatively by two pathologists, evaluating the intensity of staining $(0=$ no staining, $1=$ weak staining, $2=$ moderate staining, $3=$ strong staining) and proportion of stained cells $(0=$ no staining, $1=1-4 \%, 2=5-19 \%, 3=20-39$ $\%, 4=40-59 \%, 5=60-79 \%, 6=80-100 \%$ ). In each sample, staining of the intestinal epithelial cells was evaluated and in the case of BMAL1, the staining of intramucosal inflammatory cells was evaluated as well. The scores of intensity and proportion of staining were multiplied, yielding Quick score (QS) of 0 - 18 (18).

\section{Statistics}

The distribution of QS in IBD samples differed from normal distribution, statistical evaluation was performed using nonparametric Kruskal-Wallis test followed by Dunn's test, where the patients with $\mathrm{UC}$ and $\mathrm{CD}$ were compared mutually and consecutively with the control samples. The results were considered statistically significant when $\mathrm{p}$-value was $<0.05$.

\section{Results}

The average age of patients included in the study was 41.6 in UC group, 35.5 in CD group and 70.2 in the control group. Selected patients were predominantly of male gender; in UC group males comprised $62.5 \%$, in CD group $53.8 \%$ and $53.7 \%$ in the control group.

Immunohistochemical staining detected different expression levels of the investigated circadian rhythm-related proteins in the cytoplasm and/or the nucleus of epithelial and inflammatory cells of the intestinal mucosa. Mutual statistical comparison of QS 

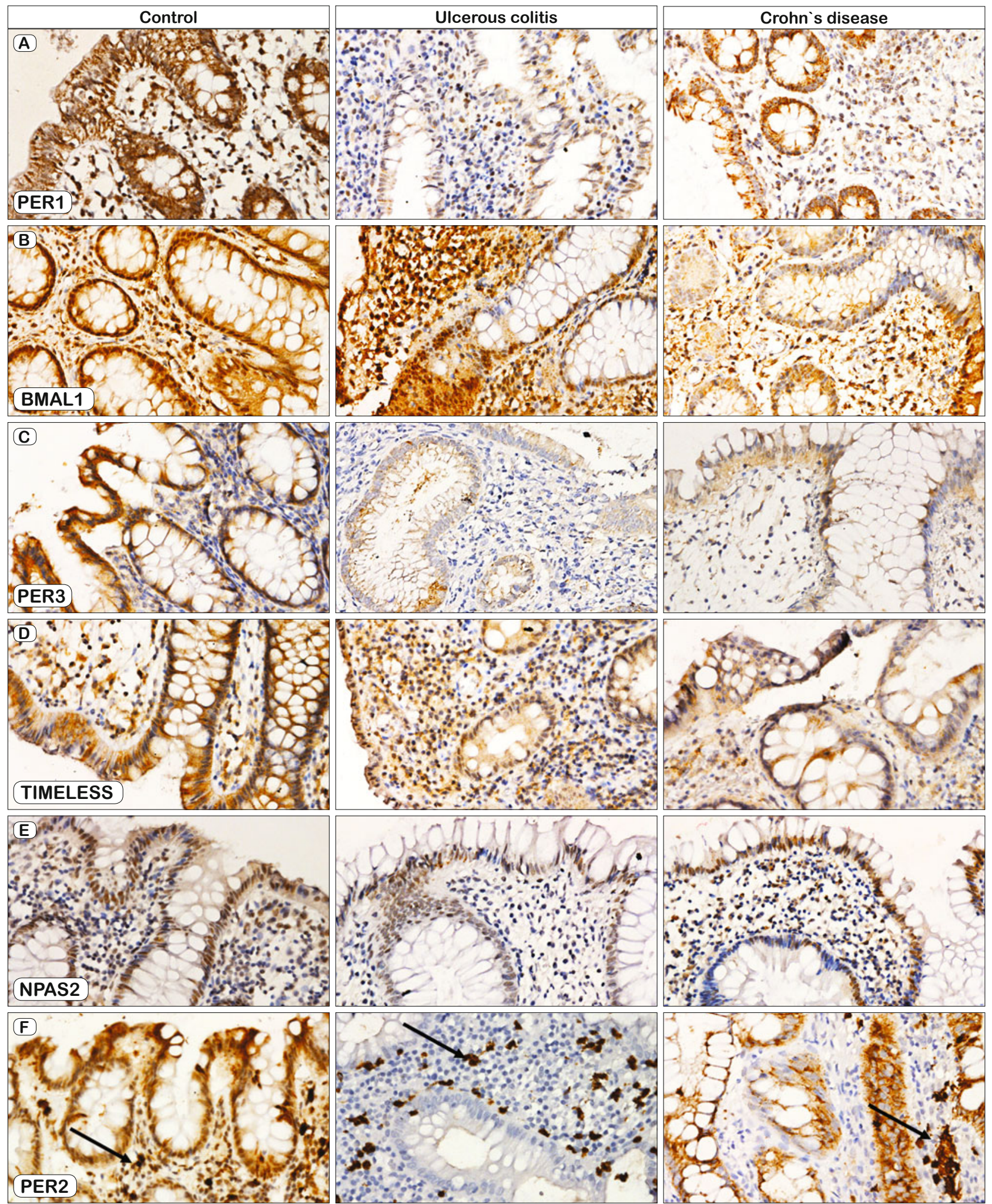

Fig. 1. Immunohistochemical detection of expression of clock proteins in intestinal mucosa of control samples compared to ulcerative colitis and Crohn's disease (A): PER1 showing combined nuclear and cytoplasmic positivity, (B): BMAL1 nuclear positivity of enterocytes and intramucosal inflammatory cells, (C): PER3 cytoplasmic positivity in enterocytes, (D): TIMELESS cytoplasmic positivity in enterocytes, (E): NPAS2 nuclear positivity in enterocytes, (F): PER2 showing areas of nuclear and/or cytoplasmic positivity with a strong combined positivity in intramucosal macrophages (arrow). Immunoperoxidase technique, diaminobenzidine, 200x. 
Tab. 1. Statistical comparison of expression of circadian proteins in patients with inflammatory bowel disease.

\begin{tabular}{|c|c|c|c|c|c|c|c|c|c|c|}
\hline & BMAL1 & $\begin{array}{c}\text { BMAL1 } \\
\text { inflammatory }\end{array}$ & BMAL2 & PER1 & $\begin{array}{c}\text { PER2 } \\
\text { cytoplasmic }\end{array}$ & $\begin{array}{l}\text { PER2 } \\
\text { nuclear }\end{array}$ & PER3 & TIMELESS & CLOCK & NPAS2 \\
\hline \multirow{2}{*}{ C vs. UC } & $* * *$ & $* * *$ & $\mathrm{~ns}$ & $* * *$ & ns & $\mathrm{ns}$ & $* *$ & $* *$ & ns & * \\
\hline & $\mathrm{P}<0.001$ & $\mathrm{P}=0.0008$ & $\mathrm{p}>0.9999$ & $\mathrm{P}<0.001$ & $\mathrm{p}>0.9999$ & $\mathrm{P}=0.4259$ & $\mathrm{P}=0.0025$ & $\mathrm{P}=0.0044$ & $\mathrm{P}=0.0979$ & $\mathrm{P}=0.0124$ \\
\hline \multirow{2}{*}{ C vs. CD } & $* * *$ & $* * *$ & $\mathrm{~ns}$ & $* * *$ & ns & $* *$ & $* * *$ & $* * *$ & ns & $* *$ \\
\hline & $\mathrm{P}<0.001$ & $\mathrm{p}<0.001$ & $\mathrm{p}>0.9999$ & $\mathrm{P}<0.001$ & $\mathrm{p}>0.9999$ & $\mathrm{P}=0.0026$ & $\mathrm{P}=0.0003$ & $\mathrm{P}<0.001$ & $\mathrm{P}=0.2496$ & $\mathrm{P}=0.0027$ \\
\hline \multirow{2}{*}{ UC vs.CD } & ns & ns & $\mathrm{ns}$ & ns & $\mathrm{ns}$ & $\mathrm{ns}$ & ns & ns & ns & ns \\
\hline & $\mathrm{P}=0.8888$ & $\mathrm{P}>0.9999$ & $\mathrm{P}=0.7209$ & $\mathrm{P}=0.5025$ & $\mathrm{P}=0.9564$ & $\mathrm{P}=0.2094$ & $\mathrm{P}<0.9999$ & $\mathrm{P}=0.9281$ & $\mathrm{p}>0.9999$ & $\mathrm{P}>0.9999$ \\
\hline
\end{tabular}

UC: Ulcerous colitis; CD: Crohn's disease; $* \mathrm{p} \leq 0.05 ; * * \mathrm{p} \leq 0.01 ; * * * \mathrm{p} \leq 0.001$. ns: not significant; : control

among samples of UC and CD patients did not show statistically significant values in any of the eight examined core circadian proteins. Statistical comparison of QS of control samples with the samples from the patients with IBD showed significant changes of expression in 5 out of 8 circadian proteins in both CD and UC (BMAL1, PER1, PER3, TIMELESS and NPAS2). Summary of the statistical evaluation of individual comparison groups is arranged in Table 1. In all of the mentioned cases, the expression of circadian proteins in enterocytes from inflamed samples was lower than in enterocytes from the control samples, whereby the most significant changes were detected in BMAL1 protein and PER1 protein, followed by PER3 and TIMELESS and the least evident changes were noted in NPAS2. Examples of histological changes of expression of these proteins in CD and UC samples in comparison to control group are demonstrated in Figure 1. Proteins PER1 and BMAL1 showed a more pronounced decrease of expression in samples from UC patients, whereas the expression of proteins PER3, TIMELESS and NPAS2 was decreased with a higher significance in the patients with $\mathrm{CD}$ than $\mathrm{UC}$, as is shown in Figure 2.

PER1 protein showed a combined cytoplasmic and nuclear expression in enterocytes and the expression was statistically sig- nificantly reduced in the intestinal samples of both $\mathrm{UC}$ and $\mathrm{CD}$ in comparison to the control samples (Fig. 2a).

Similar results were observed in BMAL1 expression within enterocytes of IBD samples, yielding a highly significant change in both UC and CD, when compared to the control samples (Fig. 1b). BMAL1 in the control samples showed a strong nuclear positivity, whereas in the samples from IBD patients, there was a clearly decreased or missing positivity in larger segments of neighbouring cells of the intestinal epithelium (Fig. 2b). Nuclear staining of intramucosal inflammatory cells showed a significant decrease in patients with UC as well as CD in comparison to the control samples (Tab. 1).

PER3 and TIMELESS proteins presented with cytoplasmic positivity in enterocytes and showed a significant decrease of expression in IBD samples, when compared to the control samples, with more pronounced changes in $\mathrm{CD}$ patients (Figs $2 \mathrm{c}, 2 \mathrm{~d}$ ).

The expression of NPAS 2 protein presented with a nuclear positivity in enterocytes with a significant loss of expression in the inflamed mucosa of both UC and CD, when compared to the control samples, with more distinct changes in CD patients (Fig. 2e).

BMAL2, CLOCK and PER2 proteins did not yield a significant difference of expression in patients with IBD, when compared to
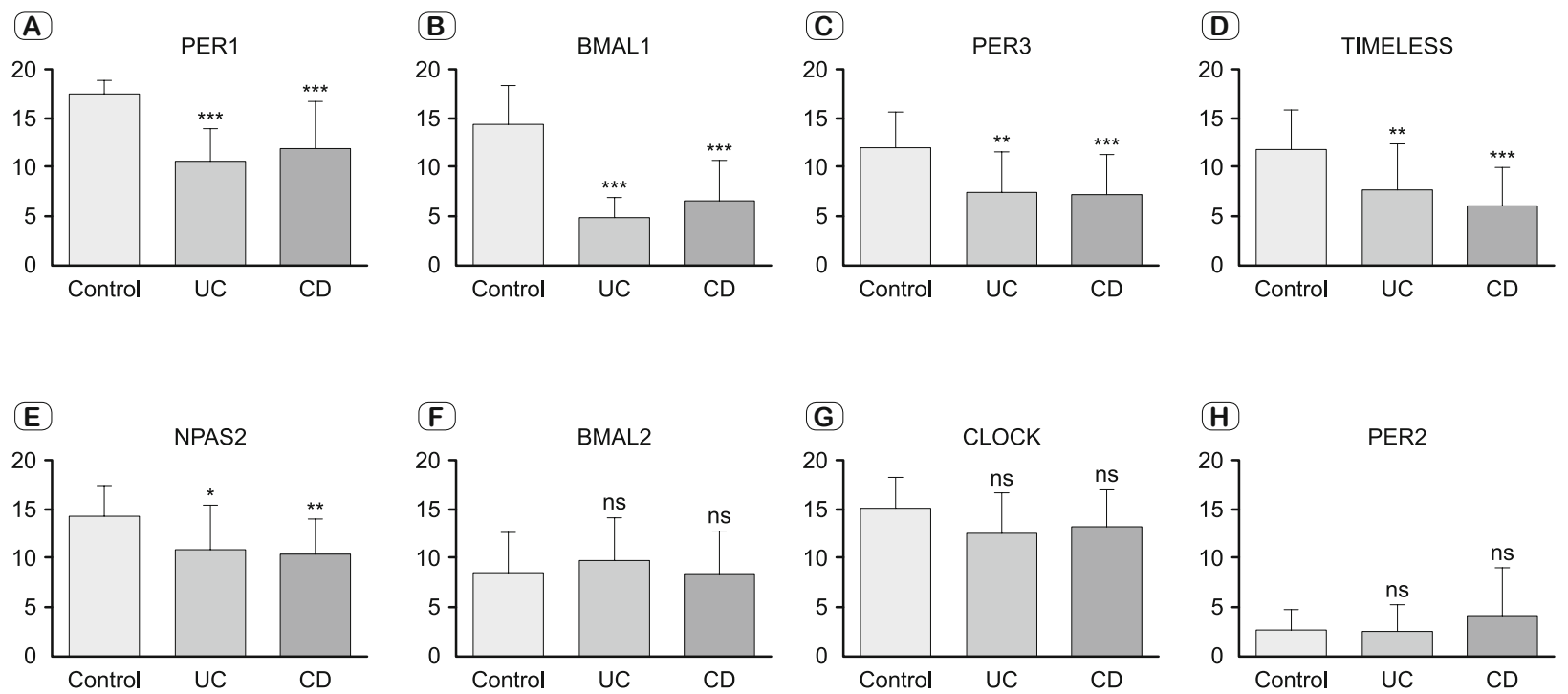

Fig. 2. Graphic form of Quick score immunohistochemical evaluation of individual circadian rhythm proteins with labeled statistical significance when comparing ulcerous colitis and Crohn's disease samples to the control. 
the controls (Tab. 1). BMAL2 and CLOCK proteins showed a predominantly diffuse cytoplasmic positivity in enterocytes, whereas PER2 protein generally showed a strong cytoplasmic positivity in isolated epithelial cells throughout the sample. Apart from this, irregular nuclear PER2 positivity was noted in intestinal epithelial cells of 13 control samples ( $52 \%$ ) which was noted in IBD samples only sporadically; in 7 cases of UC patients $(29.2 \%)$ and 2 cases of CD patients $(7.7 \%)$. In IBD patients as well as in the control group, we noted also a strong combined nuclear and cytoplasmic PER2 positivity in the macrophages (Fig. 2f). Since nuclear and cytoplasmic positivity of enterocytes showed distinct changes, we evaluated both types of expression separately. There was a significant decrease of the nuclear expression in CD patients, but an insignificant decrease in UC patients. The cytoplasmic positivity of PER2 was increased in CD patients and slightly decreased in UC patients in comparison to the control, but none of these changes proved to be significant (Tab. 1).

\section{Discussion}

Crohn's disease (CD) and ulcerative colitis (UC) are considered to be multifactorial diseases currently believed to be caused by an exaggerated immune response induced by environmental factors in genetically susceptible individuals $(2,3)$. Research over the past decade has revealed that various immune system components are under the influence of the circadian clock, manifested by daily rhythmical fluctuation in cytokine expression, recruitment of macrophages to the site of inflammation, maturation of neutrophils or phagocytosis $(19,20,21)$. Chronodisruption can thus lead to alteration of immune response, such as imbalance between pro-inflammatory and anti-inflammatory cytokines or alterations of macrophage behaviour, leading to an enhanced inflammatory state $(14,22)$. Deregulation of both innate and adaptive immune systems plays a role in the development of IBD, which might be promoted by circadian system disruption. The results of our study clearly demonstrated that although UC and CD represent two distinct clinical entities, both diseases share their connection to circadian rhythm deregulation.

The immune system also maintains bilateral interactions with gut microflora, disruption of which leads to distortion of homeostasis followed by an exacerbation of inflammation, a contributing factor in the IBD development. There is a mounting evidence that apart from stress and high-fat, high-sugar diet, the circadian system deregulation might be another promoting factor for dysbiosis development. Dysbiosis in the setting of circadian rhythm deregulation has been hypothesized to promote inflammation of GIT through an enhanced mucolytic activity leading to disruption of intestinal barrier function. Various studies subsequently confirmed differences in the composition of gut microbiota in patients with IBD versus healthy individuals (16). Recent studies pointed out, that gut microbiota affects host biological clock as well, demonstrating a reciprocal connection between the circadian rhythms and microflora $(5,23)$.

Lately, sleep and melatonin levels have been implicated in IBD development, which further testifies the impact of circadian rhythms on the gastrointestinal inflammation. Changes in the duration or quality of sleep, caused either by a shift work or lifestyle changes, affect among others the immune system through activation of NF- $\kappa \mathrm{B}$ (nuclear factor $\kappa \mathrm{B}$ ), which is responsible for the expression of pro-inflammatory genes $(5,24)$. Sleep disturbances relate to an increased rate of non-specific subjective symptoms (25), as well as an increased risk of development of various gastrointestinal disorders including IBD, where the exacerbation of the disease deteriorates sleep quality, which furthermore promotes inflammation, resulting in a self-sustaining feedback-loop $(4,26)$.

To our knowledge, up to date there were published three studies pointing out differences in the expression of clock genes in the inflamed and not inflamed intestinal mucosa (27). The first such study by Palmieri et al. conducted a genome-wide cDNA microarray analysis of 150 clock and clock-controlled genes, comparing the changes in their expression in inflamed mucosa of IBD patients with surrounding not inflamed mucosa of the same patients. This study revealed alterations of expression in more than 50 investigated genes. The core clock genes Bmal2 and Rora showed upregulation in the inflamed mucosa of CD patients while Per1, Per3 and Npas 2 were down-regulated. The inflamed mucosa of UC patients showed up-regulation of Bmal2, Cry1, Rora and Tipin genes and downregulation of Per3 (28). These results mostly correlate with our findings, showing a significantly decreased expression of PER1, PER3 and NPAS2 proteins in the inflamed mucosa and even though not significantly, but an increased expression of BMAL2 in UC patients. However, we also noted significant changes in the expression of BMAL1 and TIMELESS proteins as well.

Another study by Liu et al. compared the expression of clock genes in inflamed mucosa of IBD patients with mucosa of healthy patients. This study pointed out a significantly decreased expression of Bmal1 and Clock genes in the inflamed intestinal tissue while Bmal1 changes were more pronounced in UC patients (29). In our study, BMAL1 showed the most significantly decreased expression with more conspicuous changes in UC patients, while the expression of CLOCK protein was reduced as well, but this change was not significant. Per and Cry gene expression in the study by Liu et al. was significantly reduced in the inflamed mucosa of UC patients and with the exception of Per2 also in CD patients (29). Our results pointed out a decreased expression of PER1 and PER3 proteins in both UC and CD patients; with PER1 displaying more distinct changes in UC patients and PER3 in CD patients. We did not prove significant changes in PER 2 expression, though we noted a slight decrease of expression in UC patients and increase in CD patients.

The most recent study by Weintraub et al. evaluated the expression level of 6 core clock genes (Clock, Bmal1, Cry1, Cry2, Per1, Per2) in the intestinal mucosa of 14 newly diagnosed, previously untreated IBD patients younger than 21 years (median age 14.1). Expression levels of all clock genes were lower in the peripheral white blood cells of patients with IBD when compared to healthy individuals. They found a significantly lower expression of Clock, Bmal1, Cry1, Cry2 and Per1 in the inflamed mucosa of IBD patients and also in the non-inflamed mucosa of the same patients, 


\section{5-241}

when compared to the control group. They reported, that Per2 expression insignificantly decreased in the inflamed mucosa but significantly increased in the non-inflamed tissues of IBD patients, when compared to the control (30). We similarly proved a significant decrease of expression of PER1 and BMAL1 proteins in the epithelial cells of the inflamed mucosa of IBD patients and also a decreased expression of CLOCK protein, although not significant. The discrepancy between Per2 gene and PER2 protein expression could have been caused by the fact that this protein showed areas of either nuclear or cytoplasmic positivity, which we were evaluating separately. In our study, we evaluated the expression of PER2 protein chiefly in the intestinal epithelial cells; however, we noted a strong combined positivity of macrophages in the mucosa, which could have biased the results of gene expression. Some of the differences could be attributed to different age groups examined, (average the age of our patients was higher, 38.42) since the age is a known factor that can influence the biological clock and expression of clock genes (11). Another factor we did not take into account, when compared to the discussed study of Weintraub et al., was previous treatment, which might have attenuated the changes in intestinal epithelial cells in our cases.

Our results on circadian rhythm proteins expression generally agree with the published studies, however, currently there is only a limited amount of work focused on the investigation of changes of clock protein expression in the mucosa of IBD patients. Most of the published data dedicated to this topic analyse changes in the levels of expression of the clock genes without a discrimination between different cell types of the intestinal mucosa. Since the methodology, as well as the examined product of these two techniques differ, the results might not completely correlate (31). Considering that the evaluation of clock proteins is yet not used in the clinical setting, the concordance rate between the gene expression profiling and IHC has not been assessed so far. To our knowledge, this is so far the most extensive immunohistochemical analysis performed on samples of both UC and CD patients, which evaluated the expression of 8 different core circadian proteins in the intestinal epithelial cells. We believe, that further investigation of clock proteins expression, especially of BMAL1, PER1, PER3 and TIMELESS, which showed significant changes, when compared to the healthy mucosa, could potentially serve as diagnostic or prognostic markers of IBD patients. The changes of BMAL1 protein expression seem to be the most persuasive with an easily detectable reduction of staining in blocks of neighbouring cells, especially in UC patients.

\section{Conclusion}

Chronodisruption represents an important outcome of modern lifestyle and can play a crucial role in the course of various gastrointestinal diseases, including IBD. The results of our study are in conformity with this hypothesis and point out possible new diagnostic or prognostic markers, from which especially the BMAL1 protein seems to be the most promising. Further investigation of these proteins could bring more insight into the pathogenesis of IBD and might be useful in the management of these patients.

\section{Learning points}

- Circadian rhythm deregulation in mucosal epithelia is involved in IBD development

- Intramucosal inflammatory cells in IBD are affected by circadian rhythm deregulation

- BMAL1 protein appears to be a potent diagnostic marker of circadian system deregulation in colonic mucosa

\section{References}

1. Ng SC, Shi HY, Hamidi $\mathrm{N}$ et al. Worldwide incidence and prevalence of inflammatory bowel disease in the 21st century: a systematic review of population-based studies. The Lancet 2017; 390 (10114): 2769-2778. https: //doi.org/10.1016/s0140-6736(17)32448-0

2. Ye Y, Pang Z, Chen $\mathbf{W}$ et al. The epidemiology and risk factors of inflammatory bowel disease. Int J Clin Exp Med 2015 8 (12): 22529-22542.

3. Kim DH, Cheon JH. Pathogenesis of Inflammatory Bowel Disease and Recent Advances in Biologic Therapies. Immune Netw 2017; 17 (1): 25-40. https://doi.org/10.4110/in.2017.17.1.25.

4. Codoner-Franch P, Gombert M. Circadian rhythms in the pathogenesis of gastrointestinal diseases. World J Gastroenterol 2018; 24 (38): 4297-4303. https: //doi.org/10.3748/wjg.v24.i38.4297.

5. Sobolewska-Włodarczyk A, Wlodarczyk M, Szemraj J et al. Circadian rhythm abnormalities - Association with the course of inflammatory bowel disease. Pharmacol Rep 2016; 68 (4): 847-851. https: //doi. org/10.1016/j.pharep.2016.04.007.

6. Panda S, Hogenesh JB, Kay SA. Circadian rhythms from flies to human. Nature 2002; 417 (6886): 329-335. https: //doi.org/10.1038/417329a.

7. Hastings MH, Maywood ES, Brancaccio M. Generation of circadian rhythms in the suprachiasmatic nucleus. Nat Rev Neurosci 2018; 19 (8): 453-469. https: //doi.org/10.1038/s41583-018-0026-z.

8. Mohawk JA, Green CB, Takahashi JS. Central and Peripheral Circadian Clocks in Mammals. Annu Rev Neurosci 2012; 35: 445-462. https: //doi.org/10.1146/annurev-neuro-060909-153128.

9. Buhr ED, Takahashi JS. Molecular components of the mammalian circadian clock. Handb Exp Pharmacol 2013; 217: 3-27. https: //doi. org/10.1007/978-3-642-25950-0_1.

10. Konturek PC, Brzozowski T, Konturek SJ. Gut clock: implication of circadian rhythms in the gastrointestinal tract. J Physiol Pharmacol 2011; 62 (2): 139-150.

11. Hood S, Amir S. The aging clock: circadian rhythms and later life. J Clin Invest 2017; 127 (2): 437-446. https: //doi.org/10.1172/JCI90328.

12. Parsons MJ, Moffitt TE, Gregory AM et al. Social jetlag, obesity and metabolic disorder: investigation in a cohort study. Int J Obes 2015; 39 (5): 842-848. https: //doi.org/10.1038/ijo.2014.201.

13. Karantanos T, Theodoropoulos G, Pektasides D et al. Clock genes: Their role in colorectal cancer. World J Gastroenterol 2014; 20 (8): 1986-1992. https: //doi.org/10.3748/wjg.v20.i8.1986.

14. Scheiermann C, Kunisaki Y, Frenette P. Circadian control of the immune system. Nat Rev Immunol 2013; 13 (3): 190-198. https: //doi. org/10.1038/nri3386.

15. Matsumoto Y, Mishima K, Satoh $K$ et al. Total sleep deprivation induces an acute and transient increase in NK cell activity in healthy young volunteers. Sleep 2001; 24 (7): 804-809. 
16. Voigt RM, Forsyth CB, Green SJ et al. Circadian Disorganization Alters Intestinal Microbiota. PLoS One 2014; 9 (5): e97500. https: //doi. org/10.1371/journal.pone.0097500.

17. Magro F, Langner C, Driessen A et al. European consensus on the histopathology of inflammatory bowel disease. J Crohns Colitis 2013; 7 (10): 827-851. https: //doi.org/10.1016/j.crohns.2013.06.001.

18. Detre S, Saclani Jottim G, Dowsett M. A “quickscore” method for immunohistochemical semiquantitation: validation for oestrogen receptor in breast carcinomas. J Clin Pathol 1995; 48 (9): 876-878.

19. Scheiermann C, Kunisaki Y, Lucas D et al. Adrenergic nerves govern circadian leukocyte recruitment to tissues. Immunity 2012; 37 (2): 290-301. https: //doi.org/10.1016/j.immuni.2012.05.021.

20. Silver AC, Arjona A, Hughes ME et al. Circadian expression of clock genes in mouse macrophages, dendritic cells, and B cells. Brain Behav Immun 2012; 26 (3): 407-413. https: //doi.org/10.1016/j.bbi.2011.10.001.

21. Labrecque $\mathbf{N}$, Cermakian $\mathbf{N}$. Circadian Clocks in the Immune System. J Biol Rhythms 2015; 30 (4): 277-290. https: //doi. org/10.1177/0748730415577723.

22. Abele SH, Meadows KE, Medeiros D et al. Time is on the Immune System's Side, Yes it is. Yale J Biol Med 2019; 92 (2): 225-231.

23. Leone V, Gibbons SM, Martinez $K$ et al. Effects of diurnal variation of gut microbes and high fat feeding on host circadian clock function and metabolism. Cell Host Microbe 2015; 17 (5): 681-689. https: //doi. org/10.1016/j.chom.2015.03.006.

24. Irwin MR, Wang M, Ribeiro D et al. Sleep Loss Activates Cellular Inflammatory Signaling. Biol Psychiatry 2008; 64 (6): 538-540. https: // doi.org/10.1016/j.biopsych.2008.05.004.
25. Kosticova M, Geckova AM, Dobiasova E, Veselska ZD. Insufficient sleep duration is associated with worse self-rated health and more psychosomatic health complaints in adolescents. Bratisl Med J 2019; 120 (10): 783-788. https: //doi.org/10.4149/BLL_2019_131.

26. Graff LA, Vincent N, Walker JR et al. A population-based study of fatigue and sleep difficulties in inflammatory bowel disease. Inflamm Bowel Dis 2011; 17 (9): 1882-1889. https: //doi.org/10.1002/ibd.21580.

27. Gombert M, Carrasco-Luna J, Pin-Arboledas G et al. The connection of circadian rhythm to inflammatory bowel disease. Transl Res 2019; 206: 107-118. https: //doi.org/10.1016/j.trsl.2018.12.001.

28. Palmieri O, Mazzoccoli G, Bossa F et al. Systematic analysis of circadian genes using genome-wide cDNA microarrays in the inflammatory bowel disease transcriptome. Chronobiol Int 2015; 32 (7): 903-916. https: //doi.org/10.3109/07420528.2015.1050726.

29. Liu X, Yu R, Zhu L et al. Bidirectional Regulation of Circadian Disturbance and Inflammation in Inflammatory Bowel Disease. Inflamm Bowel Dis 2017; 23 (10): 1741-1751. https: //doi.org/10.1097/ MIB.0000000000001265.

30. Weintraub Y, Cohen S, Chapnik $\mathbf{N}$ et al. Clock Gene Disruption is an Initial Manifestation of Inflammatory Bowel Disease. Clin Gastroenterol Hepatol 2020; 18 (1): 115-122. https: //doi.org/10.1016/j.cgh.2019.04.013.

31. Fakhri GB, Akel RS, Khalil MK et al. Concordance between Immunohistochemistry and Microarray Gene Expression Profiling for Estrogen Receptor, Progesterone Receptor, and HER2 Receptor Statuses in Breast Cancer Patients in Lebanon. Int J Breast Cancer 2018; 8530318. https: // doi.org/10.1155/2018/8530318.

Received October 19, 2020. Accepted November 5, 2020 